\title{
Actualité de la jurisprudence européenne sur le temps de travail
}

\section{Recent ECJ case law on working time}

\author{
Michel Miné ${ }^{1}$
}

Published online: 28 May 2020

(C) Europäische Rechtsakademie (ERA) 2020

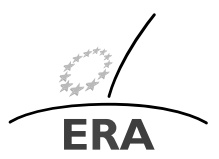

EUROPÄISCHE RECHTSAKADEMIE ACADEMY OF EUROPEAN LAW ACADEMIE DE DROIT EUROPEEN ACCADEMIA DI DIRITTO EUROPEO TRIER - TREVES - TREVIRI

Résumé Dans sa jurisprudence, le juge européen veille au respect des durées maximales du travail pour assurer la protection de la santé des travailleurs, notamment la durée maximale hebdomadaire. Il rappelle aux États membres qu'ils doivent imposer aux employeurs de mesurer les durées du travail. Le juge mobilise la Charte des droits fondamentaux pour assurer à chaque travailleur son droit aux congés annuels payés.

Mots clés Temps de travail · Santé au travail · Congés payés · Covid-19

Abstract In its case law, the European judge ensures that maximum working hours are respected to ensure the protection of workers' health, in particular the maximum weekly working time. It reminds the Member States that they must require employers to measure working hours. The judge makes use of the Charter of Fundamental Rights to ensure that every worker has the right to paid annual leave.

Keywords Working time $\cdot$ Annual leave $\cdot$ Health at work $\cdot$ Covid-19

\section{Introduction}

Le droit européen du temps de travail a pour finalité la préservation de la santé et de la sécurité des travailleurs. Cette finalité guide la Cour de justice de l'Union euro-

M. Miné, Professeur du Conservatoire national des Arts et Métiers, titulaire de la chaire Droit du travail et droits de la personne, Lise/Cnam/Cnrs.

M. Miné

michel.mine@lecnam.net

1 Conservatoire national des Arts et Métiers, 2, rue Conté - case EPN14, 75003 Paris, France 
péenne dans son interprétation téléologique des textes de l'Union. ${ }^{1}$ Ces textes sont notamment ici la Charte des droits fondamentaux de l'Union européenne, ${ }^{2}$ qui a la même valeur que les Traités, et la directive $n^{\circ}$ 2003/88/CE du Parlement européen et du Conseil du 4 novembre 2003 concernant certains aspects de l'aménagement du temps de travail. ${ }^{3}$

La question du temps de travail relève de la politique sociale : ici l'Union et les États membres disposent d'une compétence partagée. ${ }^{4}$ L'Union soutient et complète l'action des États membres dans le domaine de l'amélioration du milieu de travail, pour protéger la sécurité et la santé des travailleurs. ${ }^{5}$

Ainsi, selon la directive $\mathrm{n}^{\circ}$ 2003/88/CE, «les dispositions de la directive 89/391/CEE du Conseil du 12 juin 1989 concernant la mise en œuvre de mesures visant à promouvoir l'amélioration de la sécurité et de la santé des travailleurs au travail, ${ }^{6}$ restent pleinement applicables aux domaines couverts » par la directive sur le temps de travail, «sans préjudice des dispositions plus contraignantes et/ou spécifiques contenues dans celle-ci. » ${ }^{7}$

Au regard de cette finalité du droit de l'Union européenne, la directive rappelle que «L'amélioration de la sécurité, de l'hygiène et de la santé des travailleurs au travail représente un objectif qui ne saurait être subordonné à des considérations de caractère purement économique. ${ }^{8}$ Ce principe juridique est, de façon fort pertinente, mis en œuvre par la Cour de justice de l'Union européenne dans sa jurisprudence, concernant notamment la question essentielle de la mesure du temps de travail. ${ }^{9}$

La jurisprudence de la Cour de Luxembourg est d'une grande actualité au regard des réformes du droit du travail, décidées et mises en œuvre par des gouvernements de plusieurs États membres, avec l'aval des parlements nationaux de ces États membres, dans le cadre de la pandémie liée au coronavirus Covid-19. Le droit de l'Union fixe des limites nécessaires aux processus de régression sociale, notamment pour assurer

\footnotetext{
${ }^{1}$ Ainsi, « en appliquant le droit national, les juridictions nationales appelées à l'interpréter sont tenues de prendre en considération l'ensemble des règles de ce droit et de faire application des méthodes d'interprétation reconnues par celui-ci afin de l'interpréter, dans toute la mesure possible, à la lumière du texte ainsi que de la finalité de la directive en cause pour atteindre le résultat fixé par celle-ci », Arrêt C-55/18, du 14.05.2019, infra, spéc. §69.

${ }^{2}$ Il s'agit en particulier de l'article 31 - Conditions de travail justes et équitables 1 . Tout travailleur a droit à des conditions de travail qui respectent sa santé, sa sécurité et sa dignité. 2. Tout travailleur a droit à une limitation de la durée maximale du travail et à des périodes de repos journalier et hebdomadaire, ainsi qu'à une période annuelle de congés payés.

${ }^{3}$ JO L 299/9 du 18.11.2003, p. 1.

${ }^{4}$ TFUE, art. $2 \S 2$.

${ }^{5}$ TFUE, art. 153 , spéc. $\$ 1$ a.

${ }^{6}$ JO L 183 du 29.6.1989, p. 1.

${ }^{7}$ Dir. ${ }^{\circ}$ 2003/88/CE, considérant 3. Pour une illustration : Arrêt C-55/18, du 14.05.2019, infra, spéc. § 62.

${ }^{8}$ Dir. $n^{\circ} 2003 / 88 /$ CE, considérant 4.

${ }^{9}$ Arrêt C-266/14, du 10.09.2015, Federación de Servicios Privados del sindicato Comisiones obreras (cf. points 25, 26, 30, 32, 33, 35-39, 43, 50 et disp.), EU:C:2015:578; Arrêt C-55/18, du 14.05.2019, infra, spéc. $\$ 66$.
} 
le respect des droits fondamentaux des travailleurs. Le droit européen du Conseil de l'Europe prévoit également des bornes en la matière. ${ }^{10}$

Dans le contexte de crise sanitaire exceptionnelle, causée par la pandémie liée au Covid-19 et aux orientations défavorables des politiques publiques de santé depuis de nombreuses années dans plusieurs États-membres, la Cour de justice de l'Union européenne a rendu un important arrêt concernant le respect de la directive $n^{\circ}$ 2003/88, notamment en ce qui concerne la mesure du temps de travail et le bénéfice des temps de repos. ${ }^{11}$

Le litige concernait un policier hongrois affecté à la surveillance aux frontières extérieures de la Hongrie avec des États ne faisant pas partie de l'espace Schengen, pour empêcher l'accès de migrants au territoire national (route migratoire des Balkans). Il réclamait la requalification des temps passés en service de garde en «temps de travail ». L'employeur (la police d'intervention) estimait que la directive ne s'appliquait pas à cette activité, qui, selon lui, relevait de la dérogation prévue par la directive $\mathrm{n}^{\circ}$ $89 / 391^{12}$ (la directive $n^{\circ}$ 2003/88 y fait référence pour définir son champ d'application). ${ }^{13}$ Cette dérogation a pour objet de «préserver l'efficacité des telles activités spécifiques, dont la continuité est indispensable pour assurer l'exercice effectif des fonctions essentielles de l'État. » ${ }^{14}$

Cette affaire donne l'occasion à la Cour de préciser les conditions d'application de la dérogation, en appliquant un principe juridique essentiel : les dérogations sont d'interprétation stricte. ${ }^{15}$

Ainsi, la Cour rappelle que la directive 2003/88 s'applique à des activités dans le domaine de la santé, de la sécurité et de l'ordre publics, même lorsqu'elles sont exercées par les forces d'intervention sur le terrain et qu'elles ont pour objet de porter secours, dès lors qu'elles sont effectuées dans des conditions habituelles, conformément à la mission impartie au service concerné, et alors même que les interventions auxquelles ces activités peuvent donner lieu sont, par nature, imprévisibles et susceptibles d'exposer les travailleurs qui les exécutent à certains risques quant à leur sécurité ou à leur santé. ${ }^{16}$

Analysant l'activité en cause dans l'affaire, la Cour juge que la directive sur le temps de travail s'applique aux agents de la police d'intervention hongroise assurant la surveillance des frontières extérieures de l'État dans le contexte de fermeture de ces frontières nationales aux personnes migrantes « dans des conditions habituelles, conformément à la mission impartie $(.). » .{ }^{17} \mathrm{Il}$ en est ainsi sauf si l'activité a été

\footnotetext{
${ }^{10}$ Charte sociale européenne révisée (notamment l'art. 2) et Décisions du Comité européen des droits sociaux.

${ }^{11}$ Arrêt C-211/19, 30.04.2020, UO contre Készenléti Rendôrség, EU:C:2020:344.

${ }^{12}$ Art. 2, alinéa 1 : «La présente directive n'est pas applicable lorsque des particularités inhérentes à certaines activités spécifiques dans la fonction publique, par,exemple dans les forces armées ou la police, ou à certaines activités spécifiques dans les services de protection civile s'y opposent de manière contrai gnante. »

${ }^{13}$ Art. 1er, $\S 3$.

${ }^{14}$ Arrêt préc., $\$ 38$.

${ }^{15}$ Exceptio est strictissimae interpretationis.

${ }^{16}$ Arrêt préc., $\$ 41$.

${ }^{17}$ Arrêt préc., $\$ 47$.
} 
exercée «dans des circonstances d'une gravité et d'une ampleur exceptionnelles », relevant alors de la dérogation. Il appartiendra à la juridiction de renvoi d'examiner l'ensemble des circonstances pertinentes de cette mission de suveillance qui s'est étendue sur plusieurs mois. ${ }^{18}$

Pour la Cour de justice, l'application de la dérogation «aux services actifs dans le domaine de la santé, de la sécurité et de l'ordre publics ne se justifie qu'en raison d'événements exceptionnels, comme des catastrophes naturelles ou technologiques, des attentats ou des accidents majeurs, dont la gravité et l'ampleur nécessitent l'adoption de mesures indispensables à la protection de la vie, de la santé ainsi que de la sécurité de la collectivité, et dont la bonne exécution serait compromise si toutes les règles énoncées par la directive 2003/88 devaient être respectées. De tels cas justifient qu'une priorité absolue soit reconnue à l'objectif de protection de la population, au détriment du respect des dispositions de cette dernière directive, lesquelles peuvent provisoirement être méconnues au sein desdits services. ${ }^{19}$ Et même dans ce cas, les autorités compétentes doivent assurer la sécurité et la santé des travailleurs dans toute la mesure du possible ${ }^{20}$ (la juridiction de renvoi devra s'assurer qu'il n'était pas possible, eu égard à la gravité et à l'ampleur des circonstances, d'organiser le service concerné afin de «garantir à chaque travailleur un temps de repos conforme à ce qu'exige la directive 2003/88 $»^{21}$ ).

Dans le contexte de la crise sanitaire, l'arrêt peut permettre aux États membres de recourir à cette dérogation, pour écarter certaines dispositions de la directive sur le temps de travail, mais uniquement pour certaines catégories de personnel de la fonction publique, clairement identifiées et directement concernées. En revanche, cette dérogation ne peut être utilisée par les États-membres pour d'autres activitités publiques et a fortiori pour les activités privées ; même en période de crise sanitaire, les dispositions de la directive $n^{\circ} 2003 / 88$, telle qu'interprétée par la Cour de Luxembourg, doivent, en principe, être effectives au bénéfice de l'ensemble des travailleurs.

Ces réformes du droit du temps de travail par les États-membres doivent retenir l'attention. En effet, le droit du temps de travail est souvent utilisé comme laboratoire pour modifier le droit du travail. Ces dernières années, dans plusieurs États membres, des réformes sont allées dans le sens de la réduction des protections des salariés, concernant la stabilité de leurs horaires de travail (données qualitatives) et de l'allongement de leurs durées du travail (données quantitatives). ${ }^{22}$

Le temps de travail est au carrefour d'attentes des différents protagonistes :

- de la part des employeurs : des demandes de «flexibilité » du travail concernant les horaires de travail (transfert des risques de l'entreprise sur les salariés) et de réduction du coût du travail,

\footnotetext{
${ }^{18}$ Arrêt préc., $\$ 52$.

${ }^{19}$ Arrêt, § 42 .

${ }^{20}$ Arrêt préc., $\S 51$. Directive ${ }^{\circ} 89 / 391$, préc., art. 2 \& , alinéa 2.

${ }^{21}$ Arrêt préc., $\$ \$ 50-51$.

${ }^{22}$ M. Miné, «Quand le droit favorise l'augmentation et la flexibilité du temps de travail », La Nouvelle Revue du Travail, 11 | 2017, [4] (disponible en ligne : https://journals.openedition.org/nrt/3234).
} 
- de la part des travailleurs (salariés) : des demandes concernant la rémunération du travail, le respect et l'effectivité des droits fondamentaux de la personne, ${ }^{23}$ notamment le droit à la sauvegarde de la santé et le droit de mener une vie familiale normale, avec en particulier l'articulation entre le temps professionnel et le temps de la vie personnelle et familiale,

- de la part de la Cité : une demande de politique publique de l'emploi, la durée du travail étant un levier de cette politique, notamment pour favoriser l'emploi des travailleurs salariés et réduire le niveau du chômage (approche macro, essentiellement économique); cette demande intégrant plus ou moins bien la demande individuelle de chaque travailleur de bénéficier effectivement de son «droit au travail » (approche micro).

La jurisprudence récente de la Cour de justice de l'Union européenne porte sur la mesure de la durée du travail (2) et sur les congés payés (3).

\section{La mesure de la durée du travail}

Le droit de l'Union européenne en matière de temps de travail repose sur la définition $\mathrm{du}$ « temps de travail ». ${ }^{24}$ Cette définition donne lieu à un abondant contentieux, ${ }^{25}$ lié au non-respect de cette norme par des États membres et par des employeurs. ${ }^{26}$ La mesure du temps de travail, sur le fondement de cette définition, permet notamment d'appliquer les normes sur les repos minimums ${ }^{27}$ et sur la durée maximale du travail, au regard d'un champ d'application large concernant les activités et les travailleurs. ${ }^{28}$

\subsection{La durée maximale hebdomadaire du travail}

Selon la directive, il convient de prévoir un plafond pour la durée de la semaine de travail. ${ }^{29}$ Le texte fixe une durée maximale hebdomadaire de travail : « la durée moyenne de travail pour chaque période de sept jours n'excède pas quarante-huit heures, y compris les heures supplémentaires. » 30

\footnotetext{
${ }^{23}$ Prévus et garantis par le droit international des droits de l'homme et par le droit de l'Union européenne, notamment la Charte des droits fondamentaux.

${ }^{24}$ Dir. n $2003 / 88$, spéc. art. $2 \S 1$.

${ }^{25}$ Depuis l'arrêt C-303/98, SIMAP, 3.10.2000, EU:C:2000:528. Affaire pendante devant la Cour : C-107/19, XR contre Dopravní podnik hl. m. Prahy a.s., Concl. Av. Gral 13.02.2020, EU:C:2020:96.

${ }^{26}$ Arrêt C-14/04, A. Dellas, 01.12.2005, EU:C:2005:728. La Commission européenne n'a pas, sauf exception, engagé la procédure en manquement devant la Cour de justice de l'UE à l'encontre des États membres ne respectant pas des dispositions de la directive.

${ }^{27}$ Repos entre deux journées de travail : Arrêt C-428/09, Union syndicale Solidaires Isère,14.10.2010, EU:C:2010:612. Repos hebdomadaire : Arrêt C-306/16, Varzim Sol - Turismo, Jogo e Animacção SA, EU:C:2017:844 ; Arrêt C-102/16, Vaditrans BVBA c/ Belgische Staat, EU:C:2017:1012.

${ }^{28}$ Arrêt C-518/15, 21.02.2018, Ville de Nivelles cl Rudy Matzak, EU:C:2018:82. Arrêt C-175/16, 26.07.2017, Hannele Hälvä et autres c/ SOS-Lapsikyläry, EU:C:2017:617.

${ }^{29}$ Dir. $n^{\circ} 2003 / 88$, considérant 5.

${ }^{30}$ Dir. $n^{\circ} 2003 / 88$, art. 6.
} 
Selon la jurisprudence européenne, « la règle de la durée maximale hebdomadaire de travail de 48 heures constitue une règle du droit social communautaire revêtant une importance particulière dont doit bénéficier chaque travailleur en tant que prescription minimale destinée à assurer la protection de sa sécurité et de sa santé ». ${ }^{31} \mathrm{Et}$, par conséquent, " une réglementation nationale qui autorise des périodes de travail par semaine excédant 48 heures, y compris les services de permanence, n'apparait pas compatible avec les exigences de ladite disposition. »

La mise en œuvre effective de cette norme implique de déterminer la période de référence et d'enregistrer les durées du travail réalisées.

\subsection{La période de référence pour la mesure de la durée maximale hebdomadaire du travail - l'arrêt C-254/18 SCSI sur les périodes de référence $^{32}$}

La durée maximale hebdomadaire de travail est fixée à 48 heures. Cette durée ne s'apprécie pas au cours d'une semaine civile (du lundi 0 heure au dimanche 24 heures). Cette durée s'apprécie « en moyenne » sur une période de référence.

Pour le calcul de cette durée maximale hebdomadaire de travail, « la période de référence » peut aller jusqu'à quatre mois ${ }^{33}$ ou jusqu'à six mois par dérogation pour certaines activités, voire exceptionnellement douze mois en cas de dérogation autorisée par l'État membre. ${ }^{34}$

La question discutée concerne l'appréciation de cette période de référence de six mois. S'agit-il :

- d'une «période de référence fixe »? C'est-à-dire un semestre de l'année civile (dates calendaires fixes);

- d'une "période de référence glissante » ? C'est-à-dire une période de six mois se modifiant dont le début et la fin glissent en permanence au fil de l'écoulement du temps.

La directive est silencieuse sur cette question de l'unité de mesure du temps. Par conséquent, les États membres sont, en principe, libres de déterminer les périodes de référence selon la méthode de leur choix, sous réserve que les objectifs poursuivis par cette directive soient respectés. ${ }^{35}$

Cependant, la notion de «période de référence » est une notion autonome du droit de l'Union. ${ }^{36}$ La directive vise à garantir une protection efficace de la sécurité et de la santé des travailleurs, en prévoyant notamment une limite maximale à la durée moyenne hebdomadaire de travail. Au regard de cet objectif, « chaque travailleur doit

${ }^{31}$ Arrêt C-397/01, Pfeiffer e.a., 5.10.2004, spéc. §§ 94-95, 100-101, 120, disp. 3. Arrêt C-254/18, infra, $\S 32$.

${ }^{32}$ Arrêt C-254/18, 11.04.2019, Syndicat des cadres de la sécurité intérieure contre Premier ministre, Ministre de l'Intérieur, Ministre de l'Action et des Comptes publics, EU:C:2019:318

${ }^{33}$ Dir. $\mathrm{n}^{\circ} 2003 / 88$, art. 16 b.

${ }^{34}$ Dir. $n^{\circ}$ 2003/88, art. 19.

${ }^{35}$ Arrêt $\S \S 29$ et 31 .

${ }^{36}$ Arrêt § 24. 
notamment bénéficier de périodes de repos adéquates, qui doivent non seulement être effectives, en permettant aux personnes concernées de récupérer de la fatigue engendrée par leur travail, mais également revêtir un caractère préventif de nature à réduire autant que possible le risque d'altération de la sécurité et de la santé des travailleurs que l'accumulation de périodes de travail sans le repos nécessaire est susceptible de représenter. $»^{37}$

Concernant les dérogations admises par la directive, elles « doivent recevoir une interprétation qui limite leur portée à ce qui est strictement nécessaire pour sauvegarder les intérêts que ces dérogations permettent de protéger. » ${ }^{38}$

Ainsi, si les périodes de référence fixes et glissantes, prises isolément, sont, en soi, conformes à l'objectif de protection de la santé et de la sécurité, la combinaison de deux périodes de référence fixes successives peut, en fonction de la durée maximale hebdomadaire de travail et de la durée de la période de référence retenues par l'État membre concerné, entraîner des situations dans lesquelles cet objectif est susceptible d'être compromis. ${ }^{39}$

En effet, avec une période de référence fixe, un travailleur peut être amené à travailler, pendant une période de six mois située à cheval sur deux périodes de référence fixes successives, plus de 48 heures par semaine en moyenne. "Un tel résultat aurait pour effet de miner la dérogation prévue à cette disposition. » 40

Par conséquent, il appartient au juge national de vérifier que la réglementation nationale permet «d'assurer que la durée moyenne maximale hebdomadaire de travail de 48 heures est respectée au cours de chaque période de six mois à cheval sur deux périodes de référence fixes successives. ${ }^{41}$

Pour la Cour, les dispositions de la directive ne s'opposent pas à une réglementation nationale qui prévoit, aux fins du calcul de la durée moyenne hebdomadaire de travail, des périodes de référence qui commencent et se terminent à des dates calendaires fixes. Cependant, il en est ainsi à condition que «cette réglementation comporte des mécanismes permettant d'assurer que la durée moyenne maximale hebdomadaire de travail de 48 heures est respectée au cours de chaque période de six mois à cheval sur deux périodes de référence fixes successives. ${ }^{42}$

\subsection{L'enregistrement de la durée du travail - l'arrêt C-55/18 CCOO ${ }^{43}$}

En Espagne, une organisation syndicale, a saisi l'Audiencia Nacional (Cour centrale) d'un recours collectif dirigé contre la société Deutsche Bank, en vue d'obtenir un jugement constatant l'obligation pour cette dernière, d'établir un système d'enregistrement du temps de travail journalier effectué par les membres de son personnel,

\footnotetext{
$37 \S 34$.

38 \& 36 .

${ }^{39} \S 41$.

${ }^{40} \S 44$.

$41 \S 45$.

$42 \S 48$ et dispo.

${ }^{43}$ Arrêt C-55/18, 14.05.2019, Federación de Servicios de Comisiones Obreras (CCOO) contre Deutsche Bank SAE, EU:C:2019:402. M. Miné, «Actualité de la mesure du temps de travail », Revue de Droit du Travail, Juill.-Août 2019, p. 491, [5].
} 
permettant de vérifier le respect des horaires de travail prévus, ainsi que de l'obligation de transmettre aux représentants syndicaux les informations relatives aux heures supplémentaires effectuées mensuellement.

L'Audiencia Nacional constate notamment que Deutsche Bank utiliserait une application informatique (absences calendar) qui permettrait d'enregistrer exclusivement les absences pour une journée entière, telles que les vacances ou les autres congés, sans mesurer, en revanche, la durée du temps de travail effectué par chaque travailleur et le nombre d'heures supplémentaires réalisées. Le juge espagnol interroge la Cour de Luxembourg sur l'obligation d'établir un système d'enregistrement du temps de travail.

En Espagne, le Statut des travailleurs ne prévoyait qu'une obligation pour les employeurs d'enregistrer les heures supplémentaires effectuées par les salariés à temps plein, mais pas les heures de travail normales. Un arrêt de la Cour suprême (Tribunal Supremo) du 23 mars 2017 avait confirmé que rien n'obligeait les employeurs à décompter le temps de travail des salariés à temps plein en dehors du seul décompte des heures supplémentaires. ${ }^{44}$ Le gouvernement espagnol a anticipé l'arrêt de la Cour de justice et a adopté le décret royal $n^{\circ} 8 / 2019 .{ }^{45}$ La nouvelle disposition, entrée en vigueur le 12 mai, impose aux employeurs l'obligation générale de décompter toutes les heures de travail effectuées par les salariés. ${ }^{46}$

La décision de la Cour de Luxembourg, rendue en grande chambre, réaffirme fermement : «le droit de chaque travailleur à une limitation de la durée maximale du travail et à des périodes de repos journalier et hebdomadaire constitue non seulement une règle du droit social de l'Union revêtant une importance particulière, mais est aussi expressément consacré à l'article 31, paragraphe 2, de la Charte, à laquelle l'article 6, paragraphe 1 , TUE reconnaît la même valeur juridique que les traités ${ }^{47}$. Le premier apport de cette décision est de rappeler la nature du droit concerné ici : il s'agit d'un «droit fondamental» de la personne du travailleur ${ }^{48}$. La Charte des droits fondamentaux de l'Union européenne fait écho à des dispositions du droit international des droits de l'homme. ${ }^{49}$

En matière de temps de travail, le droit de l'Union a pour finalité la préservation de la santé de la personne au travail ${ }^{50}$. Dans cette perspective, plusieurs normes sont

\footnotetext{
${ }^{44}$ Tribunal Supremo. Sala de lo Social, $\mathrm{n}^{\circ} 81 / 2016 \mathrm{http}: / /$ www.poderjudicial.es/search/contenidos.action? action $=$ contentpdf\&databasematch $=$ TS\&reference $=7988611 \&$ statsQueryId $=114372250 \&$ calledfrom $=$ searchresults \&links=registro\&optimize $=20170411 \&$ publicinterface $=$ true

${ }^{45}$ Real Decreto-ley 8/2019, de 8 de marzo, de medidas urgentes de protección social y de lucha contra la precariedad laboral en la jornada de trabajo. https://www.boe.es/buscar/doc.php?id=BOE-A-2019-3481.

${ }^{46} \mathrm{~F}$. Javier Gomez Abelleira, professeur de droit du travail à l'université Carlos III de Madrid (entretien à IR Notes $\mathrm{n}^{\circ} 120,22$ mai 2019 - www.irshare.eu).

${ }^{47}$ Arrêt $\S 30$ et s.

${ }^{48} \S \S 31-33$.

${ }^{49}$ Not. le Pacte international des droits économiques, sociaux et culturels (PIDESC) de l'ONU (spéc. art. 7) et la Charte sociale européenne révisée du Conseil de l'Europe (spéc. art. 2).

${ }^{50}$ Dir., spéc. art. 1er, $\S 1$ («La présente directive fixe des prescriptions minimales de sécurité et de santé en matière d'aménagement du temps de travail. »). Plus largement, la directive «a pour objet de fixer des prescriptions minimales destinées à améliorer les conditions de vie et de travail des travailleurs » (Arrêt, spéc. § 36).
} 
posées, notamment la durée maximale hebdomadaire de travail et des repos minimums. Le respect de ces normes est nécessaire au regard de la finalité du droit. Pour vérifier le respect de ces normes, connaître les durées du travail réellement effectuées par chaque salarié est indispensable. La connaissance de la réalité implique la mesure des durées du travail réellement effectuées : « la détermination objective et fiable du nombre d'heures de travail quotidien et hebdomadaire est essentielle ». ${ }^{51}$ La Cour de justice de l'Union européenne réaffirme certaines obligations, d'une part, des États membres et, d'autre part, des employeurs.

Pour la Cour de Luxembourg, les États membres sont tenus de «prendre les mesures nécessaires pour que tout travailleur bénéficie » des normes protectrices énoncées dans la directive. ${ }^{52}$ Ils doivent « garantir la pleine effectivité de la directive ». ${ }^{53}$ Par conséquent, « (...) afin d'assurer l'effet utile des droits prévus par la directive 2003/88 et du droit fondamental consacré à l'article 31, paragraphe 2, de la Charte, les États membres doivent imposer aux employeurs l'obligation de mettre en place un système objectif, fiable et accessible permettant de mesurer la durée du temps de travail journalier effectué par chaque travailleur. » ${ }^{54}$

L'instauration d'un tel système «relève de l'obligation générale, pour les États membres et les employeurs, prévue à l'article 4, paragraphe 1, et à l'article 6, paragraphe 1 , de la directive $89 / 391$, de mettre en place une organisation et les moyens nécessaires pour protéger la sécurité et la santé des travailleurs (...). » 55

Cette affirmation est bienvenue dans un contexte où de plus en plus de travailleurs sont soumis à des obligations contractuelles «de résultat» imposant trop souvent des durées du travail excessives, causes de dégradation de la santé allant parfois jusqu'à l'épuisement professionnel (burn-out) ${ }^{56}$ La Cour rappelle ici aux employeurs, notamment à ceux qui fixent aux travailleurs des objectifs, qu'ils ne peuvent se désintéresser de la mesure des durées du travail de ces travailleurs.

La Cour rappelle aux États membres qu'il leur appartient de définir « les modalités concrètes de mise en œuvre d'un tel système, en particulier la forme que celui-ci doit revêtir, et cela en tenant compte, le cas échéant, des particularités propres à chaque secteur d'activité concerné, voire des spécificités de certaines entreprises (...) ».57

A contrario, une réglementation nationale qui ne prévoit pas l'obligation de recourir à un instrument permettant une détermination objective et fiable du nombre d'heures de travail quotidien et hebdomadaire n'est pas à même de garantir l'effet utile des droits conférés par la Charte et par la directive, dans la mesure où elle prive tant les employeurs que les travailleurs de la possibilité de vérifier si ces droits sont

\footnotetext{
$51 \S 49$.

$52 \S 38$.

$53 \S 40$.

${ }^{54} \S 42$ et $\S 60$.

$55 \S 62$.

56 "Long working hours and risk of coronary heart disease and stroke", The Lancet, Vol 386, October 31, 2015, p. 1739. https://www.thelancet.com/action/showPdf?pii=S0140-6736\%2815\%2960295-1

$57 \S 63$.
} 
respectés et est ainsi susceptible de compromettre l'objectif de la directive consistant à assurer une meilleure protection de la sécurité et de la santé des travailleurs. ${ }^{58}$

La Cour reprécise aux juridictions nationales leurs missions en la matière. ${ }^{59} \mathrm{La}$ décision du juge de l'Union doit conduire le juge interne à interpréter les dispositions de la directive « en tenant compte de l'importance du droit fondamental de chaque travailleur à une limitation de la durée maximale du travail et à des périodes de repos journalier et hebdomadaire. ${ }^{60} \mathrm{Et}$ « L'exigence d'une interprétation conforme inclut l'obligation, pour les juridictions nationales, de modifier, le cas échéant, une jurisprudence établie si celle-ci repose sur une interprétation du droit national incompatible avec les objectifs d'une directive $»{ }^{61}$

Le juge de Luxembourg souligne également qu'en l'absence d'un système d'enregistrement les organes de contrôle tels que l'inspection du travail sont privés d'un « moyen efficace d'obtenir l'accès à des données objectives et fiables concernant la durée du temps de travail effectué par les travailleurs dans chaque entreprise, qui s'avérerait nécessaire pour exercer leur mission de contrôle et, le cas échéant, pour infliger une sanction $\gg .62$

Concernant la situation individuelle des personnes, pour la Cour, dans une approche pragmatique, « le travailleur doit être considéré comme la partie faible dans la relation de travail, de sorte qu'il est nécessaire d'empêcher que l'employeur ne dispose de la faculté de lui imposer une restriction de ses droits » et «compte tenu de cette situation de faiblesse, un travailleur peut être dissuadé de faire valoir explicitement ses droits à l'égard de son employeur, dès lors, notamment, que la revendication de ceux-ci est susceptible de l'exposer à des mesures prises par ce dernier de nature à affecter la relation de travail au détriment de ce travailleur ». ${ }^{63}$

La Cour relève qu' « un travailleur peut (... ) recourir à d'autres moyens de preuve, tels que, notamment, des témoignages, la production de courriers électroniques ou la consultation de téléphones portables ou d'ordinateurs, afin de fournir l'indice d'une violation de ces droits $(. .$.$) ». « Toutefois, contrairement à un système mesurant la$ durée du temps de travail journalier effectué, de tels moyens de preuve ne permettent pas d'établir de manière objective et fiable le nombre d'heures de travail quotidien et hebdomadaire effectuées par le travailleur. $»{ }^{64}$ «En revanche, un système permettant de mesurer la durée du temps de travail journalier effectué par les travailleurs offre à ces derniers un moyen particulièrement efficace pour accéder de manière aisée à des données objectives et fiables concernant la durée effective du travail réalisé par eux $(. .). » .{ }^{65}$ Ainsi, « (... ) la qualification d'heures « supplémentaires » suppose que

\footnotetext{
${ }^{58} \S 50$ et dispo.

${ }^{59} \S \S 68-70$.

${ }^{60} \$ 33$.

$61 \S 70$.

$62 \S 57$.

$63 \S \S 44-45$.

$64 \S 54$.

$65 \S 56$.
} 
la durée du temps de travail effectué par chaque travailleur concerné soit connue, et donc préalablement mesurée $(. .). »{ }^{66}$

\section{Les congés payés}

Le droit aux congés annuels payés constitue un droit fondamental pour tout travailleur. Ce droit est garanti par le droit international des droits de l'homme, notamment par le Pacte international relatif aux droits économiques, sociaux et culturels ${ }^{67}$ et par des Conventions de l'Organisation internationale du travail. ${ }^{68}$

En droit de l'Union européenne, ce droit fondamental est prévu par la Charte des droits fondamentaux de l'Union européenne, ${ }^{69}$ et par la directive $\mathrm{n}^{\circ}$ 2003/88/CE ${ }^{70}$.

Selon une jurisprudence constante de la Cour de justice de l'UE, se référant notamment aux instruments internationaux ${ }^{71}$, le droit au congé annuel payé de chaque travailleur doit être considéré comme un principe du droit social communautaire revêtant une importance particulière, auquel il ne saurait être dérogé et dont la mise en œuvre par les autorités nationales compétentes ne peut être effectuée que dans les limites expressément énoncées par la directive. Le travailleur doit normalement pouvoir bénéficier d'un repos effectif, dans un souci de protection efficace de sa sécurité et de sa santé. La finalité du droit au congé annuel payé est de permettre au travailleur de se reposer et de disposer d'une période de détente et de loisirs. ${ }^{72}$ Les durées des congés payés doivent être rapprochées dans le progrès. ${ }^{73}$

\subsection{Le droit aux congés payés du salarié en arrêt de travail pour maladie}

Selon la jurisprudence de la Cour de justice de $1^{\prime} \mathrm{UE}^{74}$, tout travailleur bénéficie d'un congé annuel payé, aucune dérogation n'étant prévue par la directive : il en est ainsi quel que soit l'état de santé du travailleur. Ainsi, le droit au congé conféré au travailleur n'est pas subordonné à une prestation effective de travail préalable : le droit au congé annuel payé conféré par la directive 2003/88 à tous les travailleurs ne peut

\footnotetext{
$66 \S 52$.

${ }^{67}$ PIDESC, spéc. art. 7 : «Les États parties au présent Pacte reconnaissent le droit qu’a toute personne de jouir de conditions de travail justes et favorables, qui assurent notamment : (...) d) Le repos, les loisirs, la limitation raisonnable de la durée du travail et les congés payés périodiques, ainsi que la rémunération des jours fériés. »

${ }^{68}$ Not. les Conventions $n^{\circ} 52$ (1936) et $n^{\circ} 132$ (1970).

${ }^{69} \mathrm{Il}$ s'agit en particulier de l'article 31 - Conditions de travail justes et équitables spéc. $§ 2$ : «Tout travailleur a droit à (...) une période annuelle de congés payés. »

${ }^{70}$ Art. 7.

${ }^{71}$ Arrêt grande chambre C-350/06 et C-520/06, infra, spéc. § 38. Arrêt C-414/16, Egenberger, 17.04.2018, EU:C:2018:257, spéc. $\$ 75$.

${ }^{72}$ Arrêt grande chambre C-350/06 et C-520/06, infra.

${ }^{73}$ Arrêt C-173/99, BECTU, 26.06.2001, EU:C:2001:356, spéc. § 39.

${ }^{74}$ Depuis 1'Arrêt grande chambre C-350/06 et C-520/06, 20.01.2009, Gerhard Schultz-Hoff contre Deutsche Rentenversicherung Bund et Stringer e.a. contre Her Majesty's Revenue and Customs, EU:C:2009:18; Revue de Droit du Travail 2009.170, obs. M. Véricel.
} 
pas être subordonné par un État membre à l'obligation d'avoir effectivement travaillé pendant la période de référence établie par ledit État. ${ }^{75}$

Les applications de cette jurisprudence sont multiples; il s'agit notamment des suivantes : droit au report du congé annuel non pris du fait d'un arrêt de travail pour maladie $^{76}$ (notamment en cas de maladie au cours de la période de congés ${ }^{77}$ ), acquisition de droits à congés pendant la période d'arrêt de travail pour maladie (dans une certaine limite $\left.{ }^{78}\right)$; droit à une indemnité financière en cas d'impossibilité de prise de ce congé (à la fin de la relation de travail ${ }^{79}$ ).

\subsection{L'effectivité du droit au sein des États membres - l'arrêt C-609/17 TSN sur les congés payés et la suspension du contrat de travail pour maladie ${ }^{80}$}

Dans plusieurs États membres, le droit interne n'est pas en conformité avec le droit de l'Union européenne. Le rôle du juge interne est essentiel pour rendre effectif le droit européen au bénéfice du travailleur. Ce rôle est d'autant plus important du fait de l'inaction de la Commission à l'égard des États membres défaillants.

Comme l'avait rappelé la Cour de justice de l'UE, en cas de recours contentieux : ${ }^{81}$

- le juge interne interprète le droit interne à la lumière du droit européen pour atteindre le résultat fixé par ce droit (interprétation téléologique du droit); il s'agit en particulier de l'octroi d'une période de congés payés au salarié qui a été en arrêt de travail pour maladie au cours de la période de référence; mais il ne peut aller jusqu'à une interprétation contra legem;

- dans les entreprises liées à l'État (entreprises publiques, entreprises assurant un service public, etc.), la jurisprudence européenne doit être appliquée par le juge interne, y compris contra legem;

- à défaut, le travailleur peut engager la responsabilité de l'État.

Cependant, cette construction jurisprudentielle a été sensiblement modifiée par la Cour de Luxembourg. En effet, le juge de l'Union mobilise désormais la Charte pour rendre le droit européen efficace et en assurer l'effectivité au bénéfice des travailleurs, ses destinataires.

\footnotetext{
$75 \S 41$.

${ }^{76}$ Arrêt C-78/11, Asociación Nacional de Grandes Empresas de Distribución (ANGED) contre Federación de Asociaciones Sindicales (FASGA), Federación de Trabajadores Independientes de Comercio (Fetico), Federación Estatal de Trabajadores de Comercio, Hostelería, Turismo y Juego de UGT, Federación de Comercio, Hostelería y Turismo de CC.OO., 21.06.2012, EU:C:2012:372.

${ }^{77}$ Arrêt C-277/08, Vicente Pereda c. Madrid Movilidad SA, 10.09.2009, EU:C:2009:542; Revue de Droit du Travail 2009. 725, obs. M. Véricel. Affaire pendante : C-762/18 et C-37/19, concl. Av. fral 29.01.2020, EU:C:2020:49.

${ }^{78}$ Arrêt C-214/10, 22.11.2011, KHS AG cl M. Schulte, EU:C:2011:761.

${ }^{79}$ Arrêts C 569/16 et C 570/16, Stadt Wuppertal contre Maria Elisabeth Bauer et Volker Willmeroth contre Martina Broßonn, 06.11.2018, EU:C:2018:872.

${ }^{80}$ Arrêt C-609/17 et C-610/17, Terveys- ja sosiaalialan neuvottelujärjestö (TSN) et Auto-ja Kuljetusalan Työntekijäliitto (AKT), 19.11.2019, EU:C:2019:981.

${ }^{81}$ Arrêt grande chambre C-282/10, 24.01.2012, Maribel Dominguez contre Centre informatique du Centre Ouest Atlantique et Préfet de la région Centre, EU:C:2012:33 [1-3].
} 
Selon sa nouvelle jurisprudence ${ }^{82}$, «Le droit à une période de congés annuels payés, consacré dans le chef de tout travailleur par l'article 31, paragraphe 2, de la Charte, revêt ainsi, quant à son existence même, un caractère tout à la fois impératif et inconditionnel»; ainsi, cette disposition de la Charte «se suffit à elle-même pour conférer aux travailleurs un droit invocable en tant que tel, dans un litige qui les oppose à leur employeur $» .^{83}$

Par conséquent, « En cas d'impossibilité d'interpréter une réglementation nationale (... ) de manière à en assurer la conformité avec l'article 7 de la directive 2003/88 et l'article 31, paragraphe 2, de la Charte des droits fondamentaux », il incombe au juge interne, notamment à la juridiction de renvoi, «d'assurer, dans le cadre de ses compétences, la protection juridique découlant de ladite disposition et de garantir le plein effet de celle-ci en laissant au besoin inappliquée ladite réglementation nationale. ${ }^{84}$

Le juge interne doit faire une interprétation contra legem du droit interne afin de faire prévaloir la primauté du droit de l'Union européenne ; le juge interne doit assurer l'efficacité du droit européen pour en assurer l'effectivité au bénéfice de ses destinataires, les travailleurs. Le travailleur peut, pour obtenir, devant le juge judiciaire, le bénéfice d'une période de congés payés, qui lui a été refusé par son employeur du fait d'un arrêt de travail pour maladie, se fonder sur la Charte, prévoyant ce droit fondamental, concrétisé par la directive. Cette jurisprudence constitue à l'adresse des États membres une forte incitation à mettre en conformité leur droit interne avec le droit de l'Union ${ }^{85}$.

Cette jurisprudence s'applique au regard du droit à congé payé tel qu'il est prévu par la directive $\mathrm{n}^{\circ} 2003 / 88$.

Bien entendu, la directive 2003/88, qui fixe des « prescriptions minimales » de sécurité et de santé en matière d'aménagement du temps de travail, ne porte pas atteinte à la faculté des États membres d'appliquer des dispositions nationales plus favorables à la protection des travailleurs. Ainsi, des dispositions internes peuvent accorder un droit au congé annuel payé d'une durée supérieure aux quatre semaines ${ }^{86}$, dans les conditions d'obtention et d'octroi fixées par le droit national.

Dans ce cadre, des conventions collectives peuvent prévoir l'octroi de jours de congé annuel payé excédant la période minimale de quatre semaines prévue à la directive, tout en excluant un report pour cause de maladie de ces jours de congé. Les droits au congé annuel payé ainsi accordés au-delà du minimum requis à l'article 7, paragraphe 1 , de la directive 2003/88 sont régis non pas par cette directive, mais par le droit national (...). » ${ }^{87}$ Et les dispositions de la Charte s'adressent aux États membres uniquement lorsqu'ils mettent en œuvre le droit de l'Union. ${ }^{88}$

\footnotetext{
${ }^{82}$ Arrêt C-684/16, 06.11.2018, Max-Planck-Gesellschaft zur Förderung der Wissenschaften eV contre Tetsuji Shimizu, EU:C:2018:874.

83 \& 74.

${ }^{84} \S 80$ et dispo.

85 tue, art. $4 \S 3$ «Les États membres prennent toute mesure générale ou particulière propre à assurer l'exécution des obligations découlant des traités ou résultant des actes des institutions de l'Union. »

${ }^{86}$ Dir., art. $7 \S 1$.

${ }^{87}$ Arrêt $\$ 35$.

${ }^{88}$ Charte, art. 51.
} 
Ainsi, lorsque les États membres accordent ou permettent aux partenaires sociaux d'accorder des droits à congé annuel payé excédant la durée minimale, prévue par la directive, ces droits (avec leurs conditions et modalités de mise en œuvre) relèvent de l'exercice de la compétence des États membres, sans être réglementés par la directive; avec cette réglementation, les États membres ne procèdent pas à une mise en œuvre de la directive. La directive 2003/88 ne s'oppose pas à des réglementations nationales et à des conventions collectives qui prévoient l'octroi de jours de congé annuel payé excédant la période minimale de quatre semaines tout en excluant un report pour cause de maladie de ces jours de congé. ${ }^{89}$

Et une telle réglementation nationale se situe en dehors du champ d'application de la Charte ${ }^{90}:$ «l'article 31, paragraphe 2, de la Charte, lu en combinaison avec l'article 51, paragraphe 1, de celle-ci, doit être interprété en ce sens qu'il n'a pas vocation à s'appliquer en présence de réglementations nationales et de conventions collectives qui prévoient l'octroi de jours de congé annuel payé excédant la période minimale de quatre semaines prévue à l'article 7, paragraphe 1 , de la directive 2003/88, tout en excluant le report pour cause de maladie de ces jours de congé. » ${ }^{91}$

Publisher's Note Springer Nature remains neutral with regard to jurisdictional claims in published maps and institutional affiliations.

\section{Bibliographie}

1. Véricel, M. : Le droit à congés payés du salarié malade face à la Cour de justice européenne et à la Cour de cassation. Nouv. Rev. Trav., 371 (2012)

2. Véricel, M. : Les congés payés entre droit social de l’Union et droit de l'Union européenne. Rev. Droit Trav., 565 (2012)

3. Véricel, M. : Congé annuel payé : la cour de cassation apporte plusieurs précisions importantes. Rev. Droit Trav., 63 (2018)

4. Miné, M. : Quand le droit favorise l'augmentation et la flexibilité du temps de travail. Nouv. Rev. Travail 11 (2017). disponible en ligne. https ://journals.openedition.org/nrt/3234

5. Miné, M. : Actualité de la mesure du temps de travail. Rev. Droit Trav., 491 (2019). Juill.-Août

\footnotetext{
${ }^{89}$ Arrêt, § 40 .

${ }^{90} \S 52$ et s.

$91 \S 55$.
} 